\title{
MATERNAL PRIMARY VERSUS NON-PRIMARY CYTOMEGALOVIRUS (CMV) INFECTION AND LONG-TERM SEQUELAE OF THEIR CONGENITALLY CYTOMEGALOVIRUS (cCMV) INFECTED CHILDREN: A SYSTEMATIC REVIEW
}

\section{Background}

Maternal primary CMV infection during pregnancy is associated with a higher individual risk of transmission to the fetus when compared to non-primary infection (40$50 \%$ versus $1-2 \%) .{ }^{1}$ However, non-primary maternal CMV infection plays a significant role in the overall incidence of CMV infection worldwide, a fact attributed to the high rates of immune women of reproductive age. ${ }^{2}$

Methods

We systematically reviewed more than 100 articles from the Medline and Scopus Database, published from January 1980 till February 2018, which described neonatal symptomatology and long-term sequelae of congenitally CMV infected infants (cCMV) and compared incidence and/or severity of hearing loss status among children born to mothers with primary and non-primary CMV infection. As far as symptomatic cCMV disease, in most studies it was defined as abnormalities on clinical examination at birth. In one study however, symptomatic cCMV infection included children with abnormalities in brain imaging. The long-term sequelae of interest included sensorineural hearing loss (SNHL) and neurologic sequelae (microcephaly, neurodevelopmental disorders, seizures). Additionally, we have sifted through the references of the eligible articles and reviews.

References

1. Pass RF, Arav-Boger R., Maternal and fetal cytomegalovirus infection: diagnosis, management, and prevention., Res. 2018 Mar 1;7:255. 2. Simonazzi G1, Curti A, Cervi F, Gabrielli L, et al., Perinatal Outcomes of Non-Primary Maternal Cytomegalovirus Infection: A 15-Year Experience. Fetal Diagn Ther. 2018;43(2):138-142.

3. Permar SR, Schleiss MR, Plotkin SA, Advancing Our Understanding of Protective Maternal Immunity as a Guide for Development of Vaccines To Reduce Congenital Cytomegalovirus Infections., J Virol. 2018 Mar 14;92(7). 4. Luck SE, Wieringa JW, Blázquez-Gamero D et al., Congenital Cytomegalovirus: A European Expert Consensus Statement on Diagnosis and Management. Pediatr Infect Dis J. 2017 Dec;36(12):1205-1213.

\section{Results}

Ten articles were eligible for our systematic review and meta-analysis.

Pooled Odds Ratio (OR) for symptomatic infants following primary vs non-primary infection was 1.33 and did not reach statistical significance $(95 \% \mathrm{Cl}[0.84 ; 2.11]$, $\mathrm{p}=0.226$, Figure 1). This indicates that whether the mother had primary CMV or nonprimary CMV infection the odds of the infant becoming symptomatic is the same.

infants (primary CMV vs non-primary CMV)

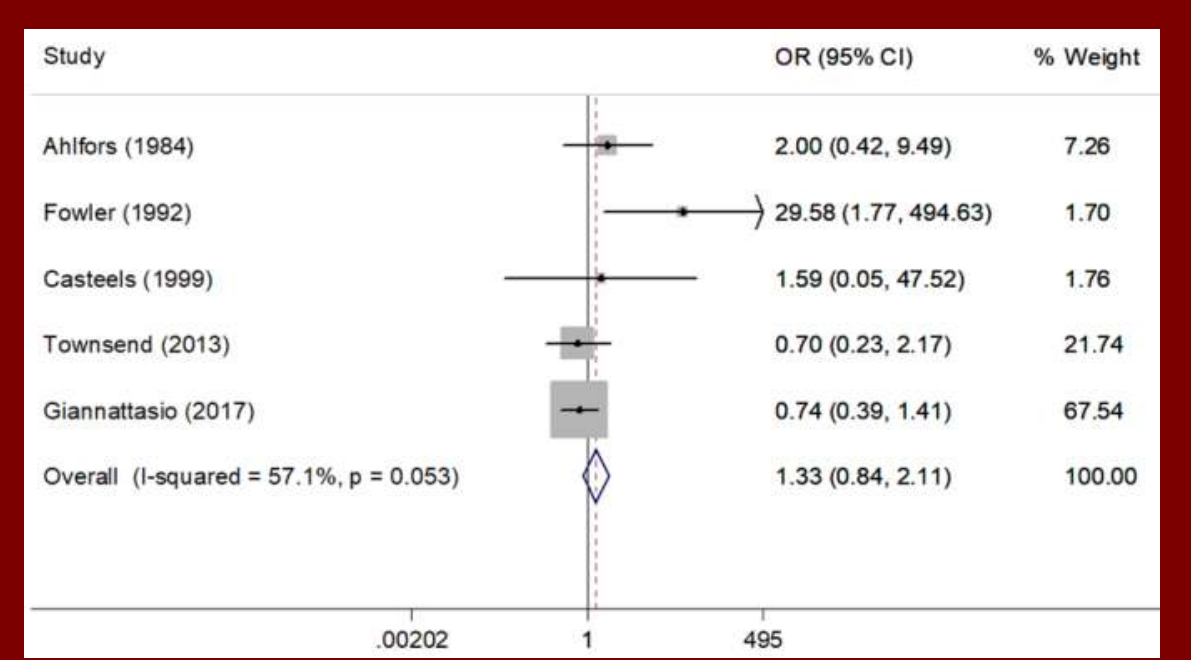

Specific outcomes were also evaluated (hearing loss, other neurologic sequelae). No statistically significant differences were detected regarding the chances of developing either outcome between the two groups. Regarding hearing loss the pooled OR between the two groups of children was 0.89 (95\% Cl [0.61-1.29], p=0.535, Figure 2).

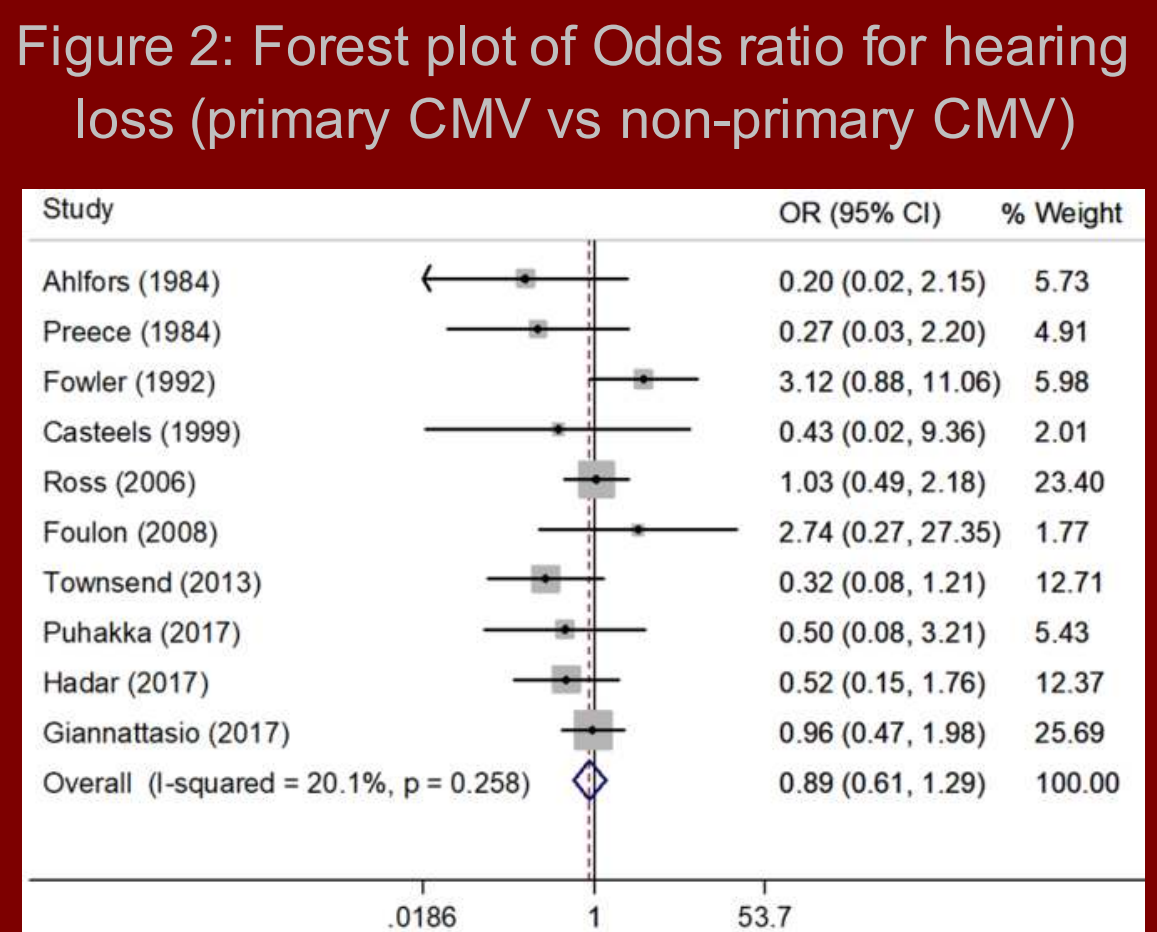

The OR for bilateral and severe hearing loss was $1.04(95 \% \mathrm{Cl}[0.53-2.07], \mathrm{p}=0.903$, Figure 3$)$ and $2.79(95 \% \mathrm{Cl}$ [0.84-9.27], $\mathrm{p}=0.094$ ) respectively.

Figure 3. Forest plot of Odds ratio for bilateral hearing loss (primary CMV vs non-primary CMV)

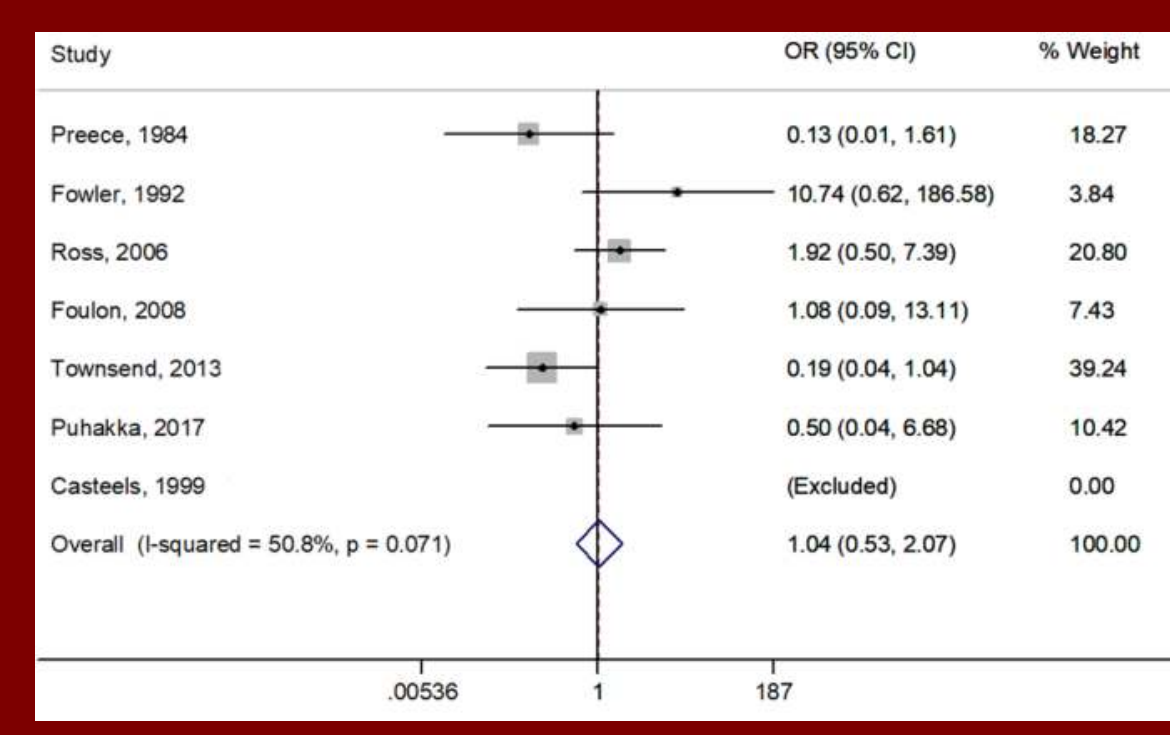

The OR for other neurologic sequelae, such as microcephaly, seizures and neurodevelopmental disorders, was $0.85(95 \%$ Cl [0.52-1.39], $p=0.518$, Figure 4).

Figure 4: Forest plot of Odds ratio for Other neurologic

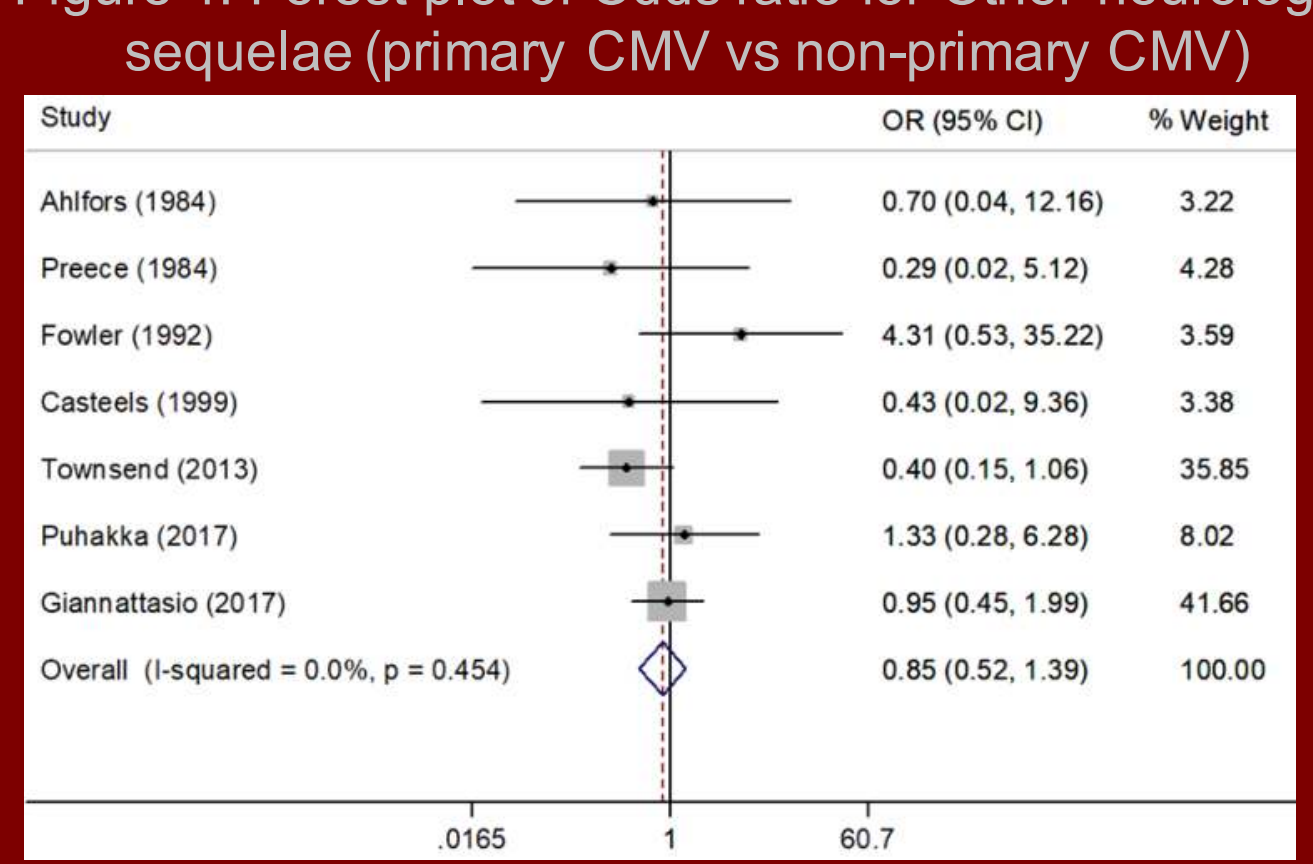

Discussion

Our systematic review and meta-analysis indicates that there is no statistically significant difference between the two groups of infants, as far as symptomatic disease at birth, hearing loss and neurologic sequelae. The important finding from the meta-analysis is that the incidence of severe/bilateral hearing loss did not depend in a statistically significant way on maternal immunity status at the time of CMV infection during pregnancy.

\section{Conclusions}

The importance of non-primary CMV infection among pregnant women is stressed. Although a recent European consensus article suggested testing only those babies born to mothers with primary CMV infection, our meta-analysis adds further support to the need, raised in this article for further research on risk factors for transmission of infection in pregnancies where mothers have been previously CMV-exposed. Furthermore, the debate on the need for a full evaluation of the potential benefits of universal screening of all newborns for CMV regardless of maternal CMV status is supported..$^{1,2}$ 